\title{
Lusioersily
}

\section{A Multi-modal Approach to Continuous Material Identification through Tactile Sensing}

Gomez Eguiluz, A., Rano, I., Coleman, SA., \& McGinnity, M. (Accepted/In press). A Multi-modal Approach to Continuous Material Identification through Tactile Sensing. In Unknown Host Publication IEEE. http://uir.ulster.ac.uk/35163/2/Decision\%20on\%20IROS\%202016\%20submission\%20142\%20\%28I\%20Rano\%2 9.docx

Link to publication record in Ulster University Research Portal

\section{Published in:}

Unknown Host Publication

Publication Status:

Accepted/In press: 01/07/2016

\section{Document Version \\ Author Accepted version}

\section{General rights}

Copyright for the publications made accessible via Ulster University's Research Portal is retained by the author(s) and / or other copyright owners and it is a condition of accessing these publications that users recognise and abide by the legal requirements associated with these rights.

\section{Take down policy}

The Research Portal is Ulster University's institutional repository that provides access to Ulster's research outputs. Every effort has been made to ensure that content in the Research Portal does not infringe any person's rights, or applicable UK laws. If you discover content in the Research Portal that you believe breaches copyright or violates any law, please contact pure-support@ulster.ac.uk. 


\title{
A Multi-modal Approach to Continuous Material Identification through Tactile Sensing
}

\author{
A. Gómez Eguíluz ${ }^{1}$, I. Rañó ${ }^{1}$, S.A. Coleman ${ }^{1}$ and McGinnity ${ }^{2}$ \\ ${ }^{1}$ Intelligent Systems Research Centre, Ulster University, Northern \\ Ireland, UK \\ ${ }^{2}$ School of Science \& Technology, Nottingham Trent University, UK
}

(C) 20xx IEEE. Personal use of this material is permitted. Permission from IEEE must be obtained for all other uses, in any current or future media, including reprinting/republishing this material for advertising or promotional purposes, creating new collective works, for resale or redistribution to servers or lists, or reuse of any copyrighted component of this work in other works. 


\title{
A Multi-modal Approach to Continuous Material Identification through Tactile Sensing
}

\author{
A. Gómez Eguíluz ${ }^{1}$ and I. Rañó ${ }^{1}$ and S.A. Coleman ${ }^{1}$ and T.M. McGinnity ${ }^{2}$
}

\begin{abstract}
Tactile sensing has recently been used in robotics for object identification, grasping, and material recognition. Most material recognition approaches use vibration information from a tactile exploration, typically above one second long, to identify the material. This work proposes a tactile multimodal (vibration and thermal) material identification approach based on recursive Bayesian estimation. Through the frequency response of the vibration induced by the material and thermal features, like an estimate of the thermal power loss of the finger, we show that it is possible to identify materials in less than half a second. Moreover, a comparison between the use of vibration only and multi-modal identification shows that both recognition time and classification errors are reduced by adding thermal information.
\end{abstract}

\section{INTRODUCTION}

Tactile sensing has recently attracted significant research interest in robotics, as a powerful way of enhancing manipulation and identification. Touch lies at the core of many human skills like grasping, material identification, and temperature detection, among others. Similarly, efforts to endow robots with tactile sensing have led to successful applications of grasping [1] and palpation [2] based object classification, object state identification [3], grasping improvement and adaptation [4] [5], and material identification [6]-[18]. This work specifically focuses on sequential material identification using multi-modal - vibration and heat - sensing. While most of the state of the art approaches to material identification through tactile sensing use only vibration signals, induced when the finger slides over the material, our experimental results show that the recognition accuracy can be enhanced using thermal information. Moreover, because our approach relies on recursive Bayesian estimation using short windows of readings, a faster material identification compared to other methods - is achieved. Thermal information also contributes to a faster material identification.

Recent research has tackled the material identification problem using vibration information obtained from different types of tactile sensors. Pioneering work in tactile surface recognition [6] used a custom built sensor with a microphone to detect vibration signals induced by textured surfaces. The authors defined a set of features to characterise the vibration signal (modal frequency and power, and average vibration amplitude). Then, they compared the classification performance using the $k$-Nearest Neighbour $(k \mathrm{NN})$ and the

\footnotetext{
${ }^{1}$ A. Gómez Eguíluz, I. Rañó, and S.A. Coleman are with the Intelligent Systems Research Centre, Ulster University, Northern Ireland, UK Gomez_Eguiluz-A@email.ulster.ac.uk \{i.rano, sa.coleman\}@ulster.ac.uk

2 T.M. Mcginnity is with the School of Science \& Technology, Nottingham Trent University, UK martin.mcginnity@ntu.ac.uk
}

K-means algorithms with the features sets and Fast Fourier Transform (FFT) coefficients, projected through Principal Component Analysis (PCA), as inputs. Their work established a methodology to classify textures, but it was not evaluated using real materials. Another material classification approach used an accelerometer based vibrotactile sensor [7]. Change in the acceleration vector (jerk) was used to create spectrotemporal histograms as features for classification. Support Vector Machine (SVM) and $k \mathrm{NN}$ were used to classify 20 materials with the features generated from several vibration readings for each material. The work in [8] presents a tactile sensor capable of measuring the strain applied on the finger surface. After preprocessing the input signal, using segmentation, average removal and band-pass filtering, the authors extract a set of features consisting of five peaks on the smoothed FFT. Combining the peak locations with the average strain readings, the authors compare different machine learning approaches that can successfully differentiate between nine materials.

In another comparison of machine learning algorithms for vibration based material identification [9], two kernel methods (SVM and Regularized Least Square), and one neural network were used to classify pairs of materials based on the raw strain measurements of the sensor. Although the authors concluded that the SVM showed the best trade-off between classification accuracy and computational complexity, the raw sensor signals provided poor discrimination performance compared with other works. In [10] a tactile micro-sensor, which can differentiate surfaces with spatial periods within a $40 \mu \mathrm{m}$ difference, is presented and used to classify textiles. A robot finger slid across the materials for two seconds, and features obtained using wavelet transforms were used in a $k \mathrm{NN}$ classifier. Through a set of temporal domain features computed from a one second signal, the work in [11] presents a SVM based classification of material texture. The sensor consists of a 3D accelerometer, and the feature vector components are whitened individually before feeding the classifier. Another accelerometer based texture recognition system is presented in [12], where the authors classify seven different fabrics based on a mixture of temporal (acceleration variance) and frequency (power spectra) features. As the textures cannot be distinguished using these features, the authors train a neural network using the FFT coefficients over a given frequency range. Recently, [13] explored real time classification of eight materials using a soft three axis tactile sensor. The vibration mean value and the Frobenius norm of its covariance matrix were fed into a cascade of binary SVMs, achieving $89 \%$ identification accuracy. 
All these pioneering works are based on custom made sensors to detect the vibration induced by a surface texture. The development of the SynTouch BioTAC fingertip which provides multiple types of tactile information opened a window of opportunity to investigate multi-modal surface recognition. A series of works [14] [15] [16], implementing human based Bayesian exploratory movements, classify materials using the BioTAC vibration, impedance, and temperature sensors. The authors identify surfaces using a combination of features measuring vibration and the friction force between the materials and the finger. If the identification certainty was not high enough the authors introduced active sensing using Bayesian inference to perform a different exploratory movement. Another multi-modal approach to material identification is presented in [17] based on readings of temperature and vibration projected through PCA. The authors show that an artificial neural network outperforms humans in similar experimental conditions. The heat transfer from a custom tactile sensor to an object is used for material identification in [18]. The authors showed that, although changes in the experiment set-up (i.e. initial conditions, ambient temperature and contact duration) have an impact on the performance, a multi-class SVM classifies 11 materials with $98 \%$ accuracy in 1.5 seconds of contact.

All these works achieve good material identification performance, however most of them do not exploit multi-modal tactile information. Moreover, they require long sequential readings, typically one second. The contribution of this work is two-fold. First, we use a recursive estimation approach with short tactile readings, which allows fast, under 0.5 secs on average, and very accurate material identification using vibration signals. Second, we show that including temperature information significantly reduces the time needed to identify the correct material and the number of classification errors. Our approach generates sequences of estimates of the posterior material probabilities instead of a single decision, and the material with the highest probability is considered the right one. The rest of the paper is organised as follows. Section II presents the methodology used for the proposed online material identification approach, including the feature generation from the raw data, probabilistic modelling, and the recursive classification technique. Experimental results comparing vibration and multi-modal material identification approaches are presented in Section III. Section IV concludes the paper and highlights future research steps.

\section{Multi-Modal Recursive Material IDENTIFICATION}

We use the SynTouch BioTAC [19] finger tip as our experimental platform. It provides a variety of sensing modalities, pressure, vibration, temperature, heat flux and finger skin deformation, with different frequencies. The finger consists of a rigid core where the sensors are located, covered by a wrinkled rubber skin. The core has a heating system and a thermistor to read the temperature of the finger. A gel separates the core from the skin. An array of impedance sensors at the core measure the deformation of the skin in contact with the materials. The rest of this section will present datails on how the vibration and thermal information is processed in our recursive Bayesian estimation framework. In a nutshell, our approach processes the vibration and thermal flux (together with the impedance) signals to obtain sets of features modelled as mixtures of Gaussians for each material. The recursive Bayesian estimation algorithm uses these models for continuous identification of the probability of each material.

\section{A. Vibration Signal Processing}

When the BioTAC slides over a surface, the interaction with the rubber wrinkles produces vibration in the skin which is transferred to the fluid, and measured by the pressure sensor. This signal is low pass filtered to generate a pressure measurement, and band pass filtered to generate a vibration signal. The vibration induced by the interaction of the wrinkled rubber skin and the texture of the materials can be seen as a combination of oscillatory signals with the frequency spectrum dependent on the material. Instead of defining features in the frequency or temporal domains, we directly use the Fourier Transform (FT) of the vibration signal to characterise the vibration response induced by the material texture. Specifically, the Fast Fourier Transform (FFT) algorithm was used to convert the vibration signal, $\rho(t)$ into the frequency domain $\rho(\omega)$. According to the existing literature [6] good discrimination results are obtained by restricting the FFT to a range of frequencies between $2 \mathrm{~Hz}$ and $500 \mathrm{~Hz}$. It is worth stating that we compute the FFT for small $(0.25 \mathrm{secs})$ non-overlapping windows of the signal of duration $\Delta t$, in order to perform online identification.

The vibration FFT $\rho(w)$ is a high dimensional vector (dimension $d_{\Delta t}$ ) of complex numbers where $d_{\Delta t}$ depends on the sampling period and the selected time interval $\Delta t$. To obtain a feature vector $\bar{\rho} \in \Re^{d}$ with a lower dimension, Principal Component Analysis (PCA) was applied to $\rho(\omega)$, where $\bar{\rho}$ denotes the projection of $\rho(\omega)$ and $d \ll d_{\Delta t}$. Because $\rho(\omega)$ is complex, yet the relevant information to classify the materials is in its modulus, the principal components were obtained from the modulus of the FFT. We observed, however, that better discrimination results were obtained if the centering process was performed in the complex FFT space, i.e. we applied PCA to the modulus upon centering with the complex mean.

\section{B. Thermal features}

The BioTAC sensor has a heating device and measures the temperature at the core and the heat flux leaving the finger. The thermal energy lost depends on the temperature difference between the finger and the external material, the contact area, and the thermal conductivity of the material. In fact, the thermal energy lost per unit of time (thermal power $\left.\frac{\partial E}{\partial T}\right)$ is the integral of the heat flux over the contact surface:

$$
\frac{\partial E}{\partial T}=\oint_{S} \vec{\phi} \cdot \overrightarrow{d S}
$$


where $\vec{\phi}$ is the heat flux, and the integral is computed on the contact area $S$. As in our experiments the BioTAC is warmer than the material, the flux $\vec{\phi}$ always leaves the finger, and its modulus, measured by the finger, increases with the temperature difference and the thermal conductivity of the material. Assuming that most of the energy is lost because of the temperature difference with the material (friction forces are too weak to generate enough thermal energy and the energy lost through the air is small), the thermal flux will be directed towards the surface normal. Moreover, the contact area is typically small, so we can approximate the power loss as the product of the average flux modulus $\bar{\phi}$ by the contact area. If we assume all objects to be identified are at the same (room) temperature, the temperature difference can be measured as the temperature at the BioTAC core, and therefore $\frac{\bar{\phi} A}{T}$ is a measure of the thermal conductivity of the material, where $A$ is the contact area, and $T$ is the finger core temperature.

To compute the contact area we use the 19 electrodes placed in the core under the BioTAC's skin. The electrodes measure impedance which is related to the distance between the core and the rubber skin at their corresponding locations. Upon contact, the skin deformation makes the readings in the closest electrodes decrease, and, therefore, electrodes with a negative value w.r.t. their resting level indicate contact. We approximate the contact area of each electrode $i$ as a circle of radius $r_{i}$ equal to half the distance between the electrode and its nearest neighbour. Hence, we compute the contact area of the fingertip as a weighted average of these areas:

$$
A=\sum_{i} \lambda_{i} \pi r_{i}^{2}
$$

where $\lambda_{i} \in[0,1]$ is an scale factor that depends on the value measured by each electrode. The scaling factor $\lambda_{i}$ is a piece-wise linear function of the average impedance value $\bar{e}_{i}$ of each electrode during the time interval $\Delta t$, such that at the resting level (or above) $\lambda_{i}$ is zero, and it increases to 1 for decreasing impedances up to a fixed minimum threshold $e_{m}$ (in our case $e_{m}=-400$ ), and is 1 for values below that threshold. Besides using the power loss per unit of temperature, just described, we defined two other features based on heat flux. We perform a linear regression of the thermal flux as a function of time in the $\Delta t$ interval, and use the slope and the regression error as additional features, as we experimentally found they help for material identification. Therefore, we obtained a three dimensional heat based feature vector $\theta$ for each $\Delta t$ time window.

\section{Material Recursive Bayesian Estimation}

We will denote by $M$ the discrete random variable encoding the $N$ materials to be identified, i.e. $\left\{m^{1}, m^{2}, \cdots, m^{N}\right\}$, $\mathrm{P} \in \Re^{d}$ is the random vector of vibration features (FFT projected through PCA) and $\Theta \in \Re^{3}$ the vector thermal features defined in section II-B. We estimate the respective likelihood functions of the feature vectors for each material, $p\left(\mathrm{P}=\bar{\rho} \mid M=m^{j}\right)$ and $p\left(\Theta=\theta \mid M=m^{j}\right)$, using mixtures of Gaussian distributions, i.e. Gaussian Mixture Models (GMM). The parameters of the GMMs are obtained using the Expectation-Maximisation (EM) algorithm, and the number of Gaussians was chosen for each material and each sensor modality based on the decrease of the likelihood of the training data, i.e. increasing the number of components to find the point at which the growth in the likelihood started to slow down.

At this stage we have models of the likelihood functions $p\left(\bar{\rho} \mid m^{j}\right)$ and $p\left(\theta \mid m^{j}\right)$ for the materials, which would already allow identification of the materials using a maximum likelihood (ML) classifier. Given a set of prior material probabilities $p\left(m^{j}\right)$, one could also estimate through the Bayes rule the posterior material probabilities $p\left(m^{j} \mid \bar{\rho}, \theta\right)$, and classify according to the maximum a posteriori (MAP) probability. Under the assumption of uninformative priors $p\left(m^{j}\right)=\frac{1}{N}$ for all $j$, the classification result of ML and MAP approaches are identical. However, as already stated, our aim is to identify materials online without the need to collect a long sequence of readings. Therefore we will use the feature vectors $\bar{\rho}$ and $\theta$ to iteratively obtain new probability posteriors for each material. The material prior $p\left(m_{k+1}^{j}\right)$ at time step $k+1$ will simply be the posterior from the previous iteration $p\left(m_{k}^{j} \mid \bar{\rho}_{k}, \theta_{k}\right)$.

When a material is presented to the robot hand for identification, the initial prior probabilities are distributed evenly among all materials. We obtain from the BioTAC data stream the vibration and thermal feature vectors, $\bar{\rho}_{k}$ and $\theta_{k}$, in windows of time $\Delta t$, and update the material probabilities using:

$$
p\left(m_{k}^{j} \mid \bar{\rho}_{k}, \theta_{k}\right)=\frac{p\left(\bar{\rho}_{k}, \theta_{k} \mid m_{k}^{j}\right) p\left(m_{k-1}^{j} \mid \bar{\rho}_{k-1}, \theta_{k-1}\right)}{p\left(\bar{\rho}_{k}, \theta_{k}\right)}
$$

where the normalisation constant $p\left(\bar{\rho}_{k}, \theta_{k}\right)$ can be obtained as:

$$
p\left(\bar{\rho}_{k}, \theta_{k}\right)=\sum_{i}^{N} p\left(\bar{\rho}_{k}, \theta_{k} \mid m_{k}^{i}\right) p\left(m_{k-1}^{i} \mid \bar{\rho}_{k-1}, \theta_{k-1}\right) \text { (4) }
$$

and we assume the vibration and thermal features are conditionally independent $p\left(\bar{\rho}_{k}, \theta_{k} \mid m_{k}^{j}\right)=p\left(\bar{\rho}_{k} \mid m_{k}^{j}\right) p\left(\theta_{k} \mid m_{k}^{j}\right)$, with each individual likelihood function given by the corresponding GMM model for material $m^{j}$.

In each iteration the algorithm updates the posterior probability $p\left(m_{k}^{j} \mid \bar{\rho}_{k}\right)$ for all materials, $j=1, \cdots, N$, and the material with the highest posterior can be considered to be the one presented to the robot. Alternatively, a confidence level could be defined to decide for a material only if the posterior probability is above some threshold. Instead of predicting the perceived texture in one episode, the recursive Bayesian estimation algorithm incrementally updates the probability estimate of the material. As a baseline to compare the recognition accuracy of our multi-modal approach, we first apply the recursive Bayesian estimation procedure to the vibration information (see section III) using expressions (3) and (4), without the thermal features $\theta$. 


\begin{tabular}{|c|c|c|c|}
\hline $\operatorname{Idx}$ & Material & Idx & Material \\
\hline 1 & Synthetic Green fabric & 18 & Genuine leather \\
\hline 2 & Synthetic Pink fabric 1 & 19 & Linen \\
\hline 3 & Synthetic Pink fabric 2 & 20 & Mirror \\
\hline 4 & Cardboard box & 21 & Normal paper \\
\hline 5 & Cardboard disk & 22 & Ping pong paddle 1 \\
\hline 6 & Carpet & 23 & Ping pong paddle 2 \\
\hline 7 & Rubber & 24 & Plastic \\
\hline 8 & Baize & 25 & Plastic dish \\
\hline 9 & Can of drink & 26 & Rough fabric \\
\hline 10 & Copper & 27 & Slate stone \\
\hline 11 & Cork & 28 & Sponge 1 \\
\hline 12 & $100 \%$ Cotton & 29 & Sponge 2 \\
\hline 13 & Padded envelope 1 & 30 & Leopard fabric 1 \\
\hline 14 & Padded envelope 2 & 31 & Leopard fabric 2 \\
\hline 15 & Aluminium & 32 & Watercolour paper \\
\hline 16 & Synthetic leather & 33 & Wood \\
\hline 17 & Floor tiles & 34 & Peach skin fabric \\
\hline
\end{tabular}

TABLE I

LIST OF MATERIALS USED IN THE CLASSIFICATION EXPERIMENTS

\section{EXPERIMENTAL RESULTS}

Our experimental setup consists of a turntable moved by a step motor through a set of reduction gears. The motor is controlled by an Arduino board running code to set the turning speed. The fingertip is attached to a worm drive bar moved up and down by a second motor also controlled by the Arduino board. If the bar is driven down, the BioTAC touches the material on the turn table. To gather the training and testing data-sets, we first set the speed of the turntable and moved the fingertip down until it touched the surface. After a few seconds we collect readings, continuously storing all the information provided by the BioTAC sensor running at a sampling rate of $4.4 \mathrm{KHz}$. This is the bandwidth at which the sensor provides individual readings, yet readings for pressure, temperature and impedance, have lower frequencies. For instance the vibration signal has a sampling frequency of $2.2 \mathrm{~K} \mathrm{~Hz}$, while pressure, absolute temperature, and thermal flux have frequencies of just $100 \mathrm{~Hz}$.

The materials to be identified were purposely selected to include different groups (metals, plastic, textiles...) and to contain similar textures. Table I presents a list of the materials used in this work, also shown in Figure 1. It can be seen that the data set contains materials with very different textures such as cork and a glass mirror, but also some fabrics and materials which are alike. To increase the number of materials, some of the fabrics were used from both sides of the cloth (materials 2 and 3, and materials 30 and 31). We also aimed to differentiate between genuine and synthetic leather represented by materials 16 and 18 , while materials 13 and 14 were obtained using both sides of a padded envelope, 13 being the side of the bubbles, i.e. plastic, and 14 the paper side. Other pairs correspond to both sides of the same object such as materials 6-7, 22-23 and 2829 , although their surfaces were clearly different.

\section{A. Vibration based material identification}

In our first experiment we classified the 34 different textures listed in Table I using only vibration information

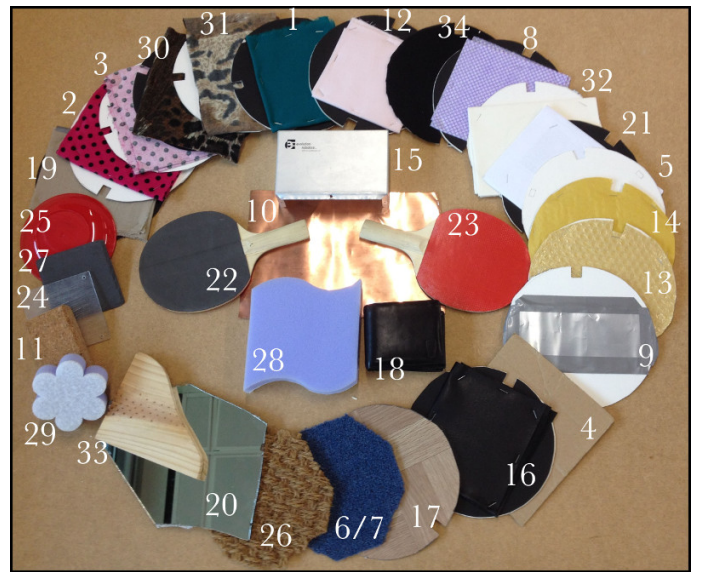

Fig. 1. Materials used in the experiments

and the approach presented in Section II. The time domain vibration signal was split into non-overlapping windows of $\Delta t=0.25$ seconds, which is therefore the minimum recognition time, and sets the frame rate for the continuous material classification to $4 \mathrm{~Hz}$. It is worth noting that, given the sampling rate and the short time interval selected $(0.25 \mathrm{sec})$, the frequency interval between spectral components is approximately $4 \mathrm{~Hz}$, that is our real minimum frequency in the band pass filter. This window corresponds to a sequence of 550 pressure samples, and upon computing the FFT and keeping the selected range of frequencies $(4-$ $500 \mathrm{~Hz}$ ) the resulting number of spectral components was 124. Increasing $\Delta t$ would provide a finer FFT approximation at the cost of reducing the classification frequency. As stated in Section II-A we reduced the 124 spectral components to a 16 dimensional space, computing the complex average of the training data-set for all 34 materials, and projecting their amplitude vectors through PCA, therefore $\bar{\rho} \in \Re^{16}$. The dimension of the reduced features was chosen to keep $97 \%$ of the total variance of the training data-set.

To evaluate our proposed approach we performed 10-fold cross validation on 5 minutes of data sequences recorded for each material. We split the data set into 10 groups of 30 seconds of sequential readings using alternatively 9 of the groups for training and one for testing. As our approach classifies the materials sequentially we used two performance measurements in the test data sequences, namely recognition errors and recognition time. For each one of the 10-fold cross-validation procedures, once the projection matrix and GMMs were obtained, the evaluation was conducted with the remaining 30 seconds time series for each material. Assuming uninformative priors and starting from the first sample the full sequence was processed, and the number of misclassifications and iterations required to successfully identify the material was stored. The first $\Delta t$ readings at the beginning of the sequence were then discarded (creating a shorter test sequence) and the process was repeated until the sequence was only of length $\Delta t$ (i.e. 0.25 seconds). This process was repeated for all the materials in the 10 testing sequences, and the average number of iterations required to 
identify each material was computed. It was considered that a material was successfully identified when the maximum posterior probability $p\left(m_{k}^{j} \mid \bar{\rho}_{k}\right)$ for that material was the highest.

As for the missclasification rate, we found that the system always properly identifies the materials when enough evidence was collected, resulting in a perfect recognition rate. However, this process can take several iterations, and, at the end of the testing sequences, the algorithm was sometimes unable to accumulate enough evidence to properly classify the textures. This leads to an average error rate of $1.21 \%$ with the errors mainly occurring in the first iteration (i.e. maximum likelihood classification).

For our second performance measurement, the recognition time, the number of iterations needed to successfully identify the material was translated into seconds multiplying by 0.25 $(\Delta t)$. Table II shows the average recognition times and standard deviations for each material over the 10 -fold crossvalidation process. As it can be seen that the vibration based recursive Bayesian estimation identifies materials within less than 0.5 seconds, and the total average time across materials (last column of the table) is approximately 1.5 iterations.

\begin{tabular}{|l|c|c|c|c|c|c|c|}
\hline Material & 1 & 2 & 3 & 4 & 5 & 6 & 7 \\
\hline mean (secs) & 0.39 & 0.35 & 0.35 & 0.43 & 0.39 & 0.3 & 0.33 \\
\hline std.dev. (secs) & 0.21 & 0.19 & 0.19 & 0.28 & 0.25 & 0.12 & 0.14 \\
\hline \hline Material & 8 & 9 & 10 & 11 & 12 & 13 & 14 \\
\hline mean (secs) & 0.44 & 0.34 & 0.3 & 0.3 & 0.35 & 0.33 & 0.34 \\
\hline std.dev. (secs) & 0.26 & 0.16 & 0.2 & 0.11 & 0.18 & 0.16 & 0.17 \\
\hline \hline Material & 15 & 16 & 17 & 18 & 19 & 20 & 21 \\
\hline mean (secs) & 0.3 & 0.44 & 0.46 & 0.32 & 0.49 & 0.29 & 0.39 \\
\hline std.dev. (secs) & 0.16 & 0.25 & 0.34 & 0.14 & 0.35 & 0.11 & 0.25 \\
\hline \hline Material & 22 & 23 & 24 & 25 & 26 & 27 & 28 \\
\hline mean (secs) & 0.38 & 0.36 & 0.38 & 0.38 & 0.27 & 0.42 & 0.29 \\
\hline std.dev (secs) & 0.22 & 0.19 & 0.22 & 0.21 & 0.07 & 0.25 & 0.13 \\
\hline \hline Material & 29 & 30 & 31 & 32 & 33 & 34 & Avg. \\
\hline mean (secs) & 0.32 & 0.27 & 0.27 & 0.46 & 0.52 & 0.4 & $\mathbf{0 . 3 6}$ \\
\hline std.dev (secs) & 0.14 & 0.08 & 0.08 & 0.3 & 0.34 & 0.26 & $\mathbf{0 . 2}$ \\
\hline
\end{tabular}

TABLE II

AVERAGE TIME NEEDED FOR MATERIAL CLASSIFICATION USING

10-FOLD CROSS VALIDATION

Figure 2 shows an example of the vibration based recursive estimation during 12 iterations ( 3 seconds) of test data for material 12 (cotton). The circle corresponds to the posterior probability of the correct material, the square corresponds to the combined probability of all other materials (i.e. excluding cotton), and the diamond corresponds to the second most likely material. The figure shows that correct identification occurs after four iterations ( 1 second). During the first two iterations there is not clear certainty on the material, in the third the posterior of material 12 is rather low. However, after the fourth iteration the classification is correct.

\section{B. Improving recognition through thermal features}

The results presented in Section III-A are our baseline to compare the multi-modal tactile approach to material identification. Therefore, in this section, we perform an identical evaluation procedure including the vibration and thermal

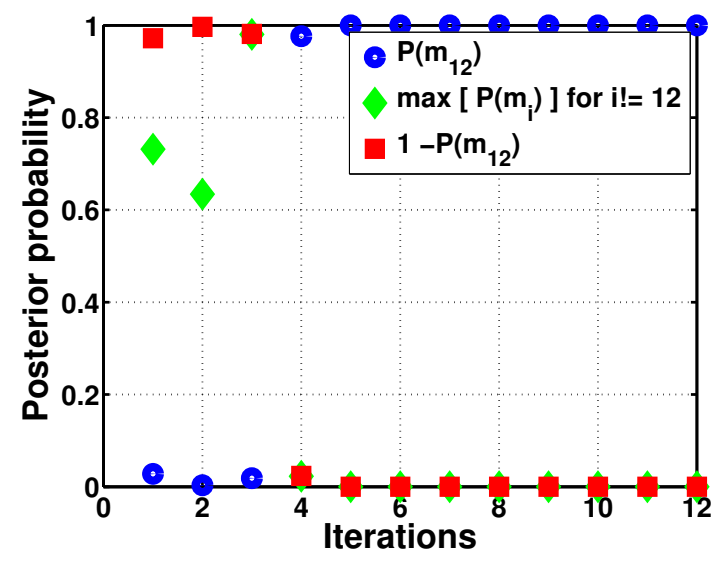

Fig. 2. Iterative estimation of material 12 using vibration

\begin{tabular}{|l|c|c|c|c|c|c|c|}
\hline Material & 1 & 2 & 3 & 4 & 5 & 6 & 7 \\
\hline mean (secs) & 0.28 & 0.28 & 0.29 & 0.28 & 0.31 & 0.26 & 0.27 \\
\hline std.dev. (secs) & 0.08 & 0.1 & 0.11 & 0.1 & 0.14 & 0.06 & 0.08 \\
\hline \hline Material & 8 & 9 & 10 & 11 & 12 & 13 & 14 \\
\hline mean (secs) & 0.33 & 0.32 & 0.26 & 0.25 & 0.28 & 0.29 & 0.26 \\
\hline std.dev. (secs) & 0.18 & 0.17 & 0.03 & 0.02 & 0.09 & 0.13 & 0.06 \\
\hline \hline Material & 15 & 16 & 17 & 18 & 19 & 20 & 21 \\
\hline mean (secs) & 0.25 & 0.38 & 0.32 & 0.30 & 0.30 & 0.26 & 0.26 \\
\hline std.dev. (secs) & 0.01 & 0.25 & 0.17 & 0.14 & 0.14 & 0.5 & 0.04 \\
\hline \hline Material & 22 & 23 & 24 & 25 & 26 & 27 & 28 \\
\hline mean (secs) & 0.27 & 0.29 & 0.27 & 0.31 & 0.25 & 0.32 & 0.25 \\
\hline std.dev (secs) & 0.07 & 0.11 & 0.07 & 0.14 & 0.01 & 0.17 & 0.01 \\
\hline \hline Material & 29 & 30 & 31 & 32 & 33 & 34 & Avg. \\
\hline mean (secs) & 0.27 & 0.26 & 0.25 & 0.29 & 0.30 & 0.26 & $\mathbf{0 . 2 8}$ \\
\hline std.dev (secs) & 0.07 & 0.06 & 0.01 & 0.13 & 0.12 & 0.06 & $\mathbf{0 . 0 9}$ \\
\hline
\end{tabular}

TABLE III

TEMPERATURE FOR RECOGNITION IMPROVEMENT. AVERAGE TIME NEEDED USING 10-FOLD CROSS VALIDATION

features $\left(\theta_{k}\right)$ described in section II-B. As already stated, the sampling frequency of the thermal flux, temperature, and impedance sensors, is lower than the vibration sampling, and our $\Delta t$ window corresponds to 25 samples of each of these signals. To estimate the thermal power loss we averaged the heat flux and impedance readings during the measuring interval, while the heat flux slope and error are computed with all the samples. The number of Gaussians in the mixture model for the thermal features was typically 2 , except for materials 4, 8, 12 and 23 which we estimated as 3 using the changes in the training data likelihood.

Our experiments showed that including thermal information reduces the average errors on the material classification with respect to vibration only classification. Specifically, the number of misclassification samples measured as explained in Section III-A becomes $1.02 \%, 15.6 \%$ reduction in the classification errors, w.r.t the vibration only classification described in the previous section. Table III shows the average and standard deviation of the classification time using the multi-modal approach. Both, the overall average time and the standard deviation are reduced (cf. Table II) which means the 


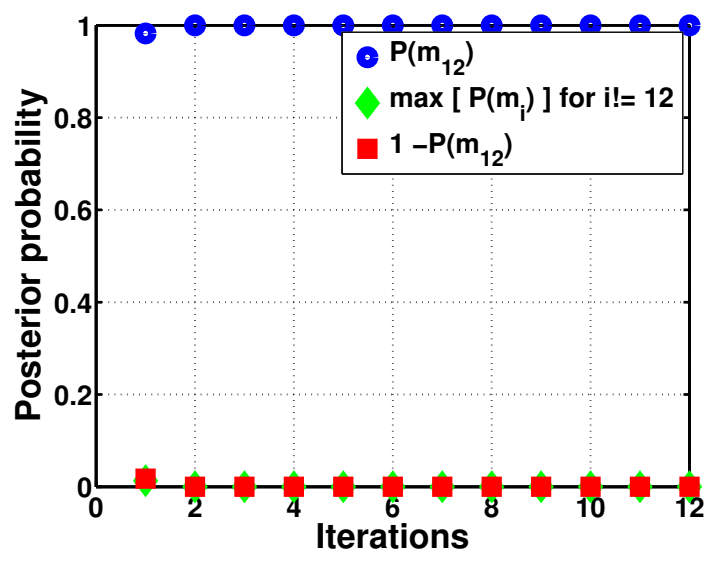

Fig. 3. Multi-modal iterative estimation of material 12.

recognition occurs faster ( $22 \%$ improvement on the average), but it is also more stable. Since the average recognition time is now 0.28 seconds and the recognition period is 0.25 seconds there is not much room for improvement, which actually means almost all of the materials are now identified in one step. While for some materials including thermal information slightly improves the recognition time (e.g. materials 30/31), in most of the cases the improvement in time is above $10 \%$, with material 33 (wood) experiencing a time recognition improvement of over $40 \%$.

Figure 3 shows the evolution of the posterior probabilities for the recognition of material 12 for the same test sequence shown in Figure 2. As it can be seen (cf. Figure 2) including thermal information makes the identification correct from the first iteration on, reducing in this case the identification time from 1 second to 0.25 seconds.

\section{CONCLuSions And Future Work}

This paper presents a multi-modal tactile based continuous material identification approach. While state of the art approaches to material identification mostly rely on vibration information, we show that including thermal features reduces the material classification errors. Moreover, standard tactile identification techniques typically require a sequence of at least one second to classify materials. Using recursive Bayesian estimation a robot endowed with tactile sensors can identify materials in an average of 0.28 seconds with a very small deviation from that time lapse. This detection speed is again due to the use of multi-modal information. Hence thermal sensing not only reduces the number of errors but also enables a faster identification than a vibration only approach. We also eliminated the need for one or several long exploratory movements for surface identification found in the related literature.

Including thermal information brings the average material identification time very close to the used window size. Faster identification could be achieved by reducing the size of the window, yet the selection of $\Delta t=0.25$ was empirically found to provide an excellent time-recognition trade-off.
Therefore, shorter time will possibly imply worse accuracy. Our future work will include other sensing modalities, specifically vision, to enhance material recognition, for instance, generating visual texture based priors. Tactile information could also generate priors for the visual recognition of objects, and, in general, these two sensing modalities could be used together in a continuous object identification system with joint visual-tactile object models.

\section{REFERENCES}

[1] N. Gorges, S. Navarro, D. Göger, and H.Wörn, "Haptic object recognition using passive joints and haptic key features," in Proc. of IEEE Intl. Conf. on Robotics and Automation, 2010, pp. 2349-2355.

[2] A. Scheneider, J. Sturm, C. Stachniss, M. Reisert, H.Burkhardt, and W. Burkhardt, "Object identification with tactile sensors using bag-offeatures," in IEEE/RSJ Intl. Conf. on Intel. Robots and Systems, 2009, pp. 243-248.

[3] S. Chitta, J. Sturm, M. Piccoli, and W. Burgard, "Tactile sensing for mobile manipulation," IEEE Trans. on Robotics, vol. 3, no. 27, pp 558-568, 2011.

[4] J. Romano, K. Hsiao, G. Niemeyer, S. Chitta, and K. Kuchenbecker, "Human-inspired robotic grasp control with tactile sensing," IEEE Trans. on Robotics, vol. 6, no. 27, pp. 1067-1079, 2011.

[5] K. Hang, M. Li, J. Stork, Y. Bekiroglu, A. Billard, and D. Kragic, "Hierarchical fingertip space for synthesizing adaptable fingertip grasps," in ICRA 2014 Workshop: Autonomous Grasping and Manipulation: An Open Challenge, 2014.

[6] J. Edwards, J. Lawry, and C. Melhuish, "Extracting textural features form tactile sensors," Bioinspiration \& Biomimetics, vol. 3, no. 3, p. 035002, 2008.

[7] J. Sinapov, V. Sukhoy, R. Sahai, and A. Stoytchev, "Vibrotactile recognition and categorization of surface textures by a humanoid robot," IEEE Trans. on Robotics, vol. 3, no. 27, pp. 488-497, 2011.

[8] N. Jamali and C. Sammut, "Majority voting: material classification by tactile sensing using surface texture," IEEE Trans. on Robotics, vol. 3 , no. 27, pp. 508-521, 2011.

[9] S. Decherchi, P. Gastaldo, R. Dahiya, M. Valle, and R. Zunino, "Tactile-data classification of contact materials using computational intelligence," IEEE Trans. on Robotics, vol. 3, no. 27, pp. 635-639, 2011

[10] C. Oddo, M. Controzzi, L. Beccai, C. Cipriani, and M. Carrozza "Roughness encoding for discrimiation of surfaces in artificial activetouch," IEEE Trans. on Robotics, vol. 3, no. 27, pp. 522-533, 2011.

[11] P. Dallaire, P. Giguère, D. Émond, and B. Chaib-draa, "Autonomous tactile perception: A combined improved sensing and bayesian nonparametric approach," Robotics and autonomous systems, no. 62, pp. 422-435, 2014.

[12] D. Chathuranga, V. Ho, and S. Hirai, "Investigation of a biomimetic fingertip's ability to discriminate fabrics based on surface textures," in 2013 IEEE/ASME Intl. Conf. on Advanced Intelligent Mechatronics. Mechatronics for Human Wellbeing, 2013, pp. 1667-1674.

[13] D. Chathuranga, Z. Wang, Y. Noh, T. Nanayakkara, and S. Hirai, "Robust real time material classification algorithm using soft three axis tactile sensor: Evaluation of the algorithm," in 2015 IEEE/RSJ Intl. Conf. on Intel. Robots and Systems, 2015.

[14] J. Fishel and G. Loeb, "Bayesian exploration for intelligent identification of textures," Frontiers in Neurorobotics, no. 6, pp. 1-20, 2012.

[15] D. Xu, G. Loeb, and J. Fishel, "Tactile identification of objects using bayesian exploration," in 2013 IEEE Intl. Conf. on Robotics and Automation, 2013, pp. 3056-3061.

[16] Z. Su, J. Fishel, T. Yamamoto, and G. Loeb, "Use of tactile feedback to control exploratory movements to characterize object compliance," Frontiers in Neurorobotics, no. 6, pp. 1-9, 2012.

[17] E. Kerr, T. Mcginnity, and S. Coleman, "Material classification based on thermal and surface texture properties evaluated against human performance," in 13th Intl. Conf. on control automation robotics \& vision, 2014, pp. 10-12.

[18] T. Bhattacharjee, J. Wade, and C. Kemp, "Material recognition from heat transfer given varying initial conditions and short-duration contact," in Proc. of Robotics: Science and Systems, 2015

[19] J. Fishel, G. Lin, and G. Loeb, "Syntouch LLC biotac product manual, v. 16," Tech. Rep., 2013. 